\title{
Hydrocarbon isotope detection by elastic peak electron spectroscopy
}

\author{
I.A. Kostanovskiy* and V.P. Afanas'ev \\ National Research University MPEI, \\ Krasnokazarmennaya 14, 111250, Moscow, Russia \\ D. Naujoks \\ Max-Planck-Institut für Plasmaphysik, Teilinstitut Greifswald, \\ Wendelsteinstraße 1, D-17491 Greifswald, Germany \\ M. Mayer \\ Max-Planck-Institut für Plasmaphysik, D-85748 Garching, Germany
}

(Dated: October 16, 2014)

\begin{abstract}
Experimental results on the hydrocarbon isotope analysis by elastic peak electron spectroscopy are presented. Amorphous hydrocarbon samples (a-C:H, a-C:D) are prepared by PCVD and analyzed by nuclear reaction analysis (NRA), elastic recoil detection analysis (ERD), X-ray photoelectron spectroscopy (XPS) and elastic peak electron spectroscopy (EPES). Electron energy spectra show a clear signal from the hydrogen isotopes deuterium and protium. Different incident energies and scattering geometries help to resolve plasmon and elastic energy losses.

Keywords: Isotope detection, NRA, ERD, EPES, XPS, Fusion
\end{abstract}




\section{INTRODUCTION}

Quantitative analysis of the hydrogen content in the near-surface layer of a sample is one of the key tasks in many studies. Implantation and deposition of hydrogen isotopes from a thermonuclear plasma, formatting PCVD coatings or developing H-storage materials - all these techniques need information on atomic or isotope depth profiles[1, 2]. Most common techniques are elastic recoil detection analysis (ERD), secondary ion mass spectrometry (SIMS) or nuclear reaction analysis (NRA) [3]. They are known to be isotope sensitive and have a depth resolution of up to $0.5 \mathrm{~nm}$. However, all of them have one well known disadvantage: these are not in-situ methods. Kimura et al. [4] performed ERD analysis of hydrocarbon CVD films and has shown, that most of the hydrogen is localized mainly in the first several monolayers. In this case in-situ analysis techniques are required, for example elastic peak electron spectroscopy (EPES).

Boersch et al. presented the idea and experimental realization of EPES for the first time in 1961 [5]. The main concept behind EPES is a well known solution for the elastic scattering of electron with the mass $m_{e}$ on the scattering atom with the mass $M_{A}$. For this case the elastic energy loss $\Delta E$ is proportional to the primary energy $E_{0}$ of the electron and the scattering angle $\theta$.

$$
\Delta E=E_{0} \frac{2 m_{e}}{M_{A}}(1-\cos \theta)
$$

Equation (1) shows that the elastic energy loss is of the order of $10 \mathrm{eV}$ for $E_{0}=30 \mathrm{keV}$ and decreases with decreasing primary energy. Therefore the most important prerequisite for this technique is high energy resolution. As a result the development of EPES started only in the recent 20 years, when high resolution energy analyzers became commercially available. A series of works by Vos et al. shows how elastic energy losses can be resolved and used to determine the mass of the scattering atom and the surface composition for thicknesses of the order of $10 \mathrm{~nm}$ [6-8]. The depth of EPES analysis can be approximated from the inelastic mean free path (IMFP) and the incident and reflection angles towards the surface normal $-\xi, \eta$.

$$
d_{\text {analysis }}=\frac{I M F P\left(E_{0}\right)}{\frac{1}{\cos \xi}+\frac{1}{\cos \eta}}
$$

Equation 2 shows that the EPES technique can be applied for IMFP determination, which is difficult by other methods [9-13]. Additionally, EPES could be applied for the analysis 
of hydrogen isotopes concentrations in polymers [14], frozen water $\left(\mathrm{D}_{2} \mathrm{O}, \mathrm{H}_{2} \mathrm{O}\right)[15]$ and also in $\mathrm{CD}_{4}$ and $\mathrm{CH}_{4}$ gases [16].

In contrast to SIMS the depth profiling by EPES does not need sputtering layer by layer. Analysis depth can be changed by changing the primary energy of electrons or by choosing another scattering geometry. Using electrons is convenient in compare to the NRA technique where the maximum incident energy and therefore the analysis depth is specified by the nuclear reaction. Taken together, the main aim of this work is to demonstrate the application of EPES for element analysis of CVD films with different isotopes like protium and deuterium, which are of main importance, for example, for plasma-wall interaction research in thermonuclear fusion devices.

\section{EXPERIMENT}

Amorphous hydrocarbon layers containing either protium $(\mathrm{CH})$ or deuterium (CD) were deposited on silicon substrates in $\mathrm{CH}_{4}$ or $\mathrm{CD}_{4}$ plasmas, respectively, at gas pressures of $2 \mathrm{~Pa}$ at a bias voltage of $-300 \mathrm{~V}$. The layer thicknesses as determined by ellipsometry were about $70 \mathrm{~nm}$ and $160 \mathrm{~nm}$, respectively. The amount of deuterium was determined by nuclear reaction analysis (NRA) using the $\mathrm{D}\left({ }^{3} \mathrm{He}, \mathrm{p}\right) \alpha$ reaction at $2.5 \mathrm{MeV}$ incident energy and a reaction angle of $135^{\circ}$. The amount of carbon was determined by NRA using the reactions ${ }^{12} \mathrm{C}\left({ }^{3} \mathrm{He}, \mathrm{p}_{0}\right){ }^{14} \mathrm{~N}$ and ${ }^{12} \mathrm{C}\left({ }^{3} \mathrm{He}, \mathrm{p}_{1}\right){ }^{14} \mathrm{~N}$ at $2.5 \mathrm{MeV}$ incident energy and the reaction angle of $135^{\circ}$. The accuracy of measurements was about $5 \%$. The amount of $\mathrm{H}$ was determined with $3 \mathrm{MeV}$ incident ${ }^{4} \mathrm{He}$ applying elastic recoil detection analysis (ERD). The SIMNRA program [17] with SigmaCalc cross-section for the hydrogen recoils [18] was used for the data evaluation. The absolute accuracy of ERDA is about $15 \%$. The sample properties are summarized in Table 1.

\begin{tabular}{ccc}
\hline & \multicolumn{2}{c}{ Concentration, atoms $/ \mathrm{cm}^{-2}$} \\
\hline & a-C:H & a-C:D \\
\hline Carbon & $6.27 \cdot 10^{17}$ & $1.20 \cdot 10^{18}$ \\
\hline Protium & $3.35 \cdot 10^{17}$ & - \\
\hline Deuterium & - & $7.53 \cdot 10^{17}$
\end{tabular}

TABLE I. Properties of the a-C:H and a-C:D layers 
Electron spectroscopy experiments were performed in ultra-high vacuum conditions $\left(\mathrm{p}=2.5 \cdot 10^{-10} \mathrm{mbar}\right)$. The standard experimental setup consists of a $\mu$-metal UHV chamber and is equipped with a Specs Phoibos 225 analyzer with 2D-CCD detector. The energy range of the analyzer is $0-15 \mathrm{keV}$ with the best resolution of $0.03 \mathrm{eV}$ at the maximum kinetic energy. The angular range of the analyzer is $+/-13^{\circ}$ and could be adjusted by an iris diaphragm. The system is equipped with a non-monochromatic X-ray source SPECS XR50 with Ag/Mn anodes. Two Kimball Physics EG-4212 electron guns can accelerate electrons up to $30 \mathrm{keV}$. They realize two scattering angles - $45^{\circ}$ for glancing geometry and $120^{\circ}$ for reflected geometry. The guns are equipped with $\mathrm{BaO}$ cathodes for minimizing of the energy spread.

The deposited samples were exposed to ambient atmosphere before being measured by electron spectroscopy. In order to save hydrogen in the surface, especially in the first several monolayers, the samples were not annealed or degassed. First information on the surface composition was received by XPS with non monochromatic Mn K $\alpha$ source.

Under the X-ray beam the pressure in the chamber raised to about $10^{-9} \mathrm{mbar}$. To avoid a surface degradation the low emission mode of the Kimball Gun was used. This keeps the pressure at $4 \cdot 10^{-10}$ mbar during the EPES measurements. The emission was set to be constant and was $50 \mathrm{nA}$ for all EPES experiments. EPES spectra have been measured with primary energies of 3,5 and $8 \mathrm{keV}$. The energy range was $50 \mathrm{eV}$ in order to include the contribution from plasmons. Such an energy range corresponds sometimes to reflected electron energy loss technique EELS. The pass energy of $40 \mathrm{eV}$ defines the energy resolution of the analyzer which was about $0.05 \mathrm{eV}$. The step size was minimized to the value of the energy resolution with a dwell time of $0.3 \mathrm{~s}$ at each step. The angular range was minimized for $+/-2^{\circ}$.

\section{RESULTS AND DISCUSSIONS}

Fig. 1-a shows the XPS spectrum of the CD sample, which contains a carbon and an oxygen peak. The shape of the spectrum for the $\mathrm{CH}$ sample (Fig. 1-b) is similar, although is has different peak intensities. Both spectra were fitted in CasaXPS software. The relative concentration of carbon is $93 \%$ for the CD sample (7\% oxygen) and $95 \%$ ( $5 \%$ oxygen) for the $\mathrm{CH}$ sample. A zoomed region with the Auger carbon peak is shown in Fig. 1-c and 

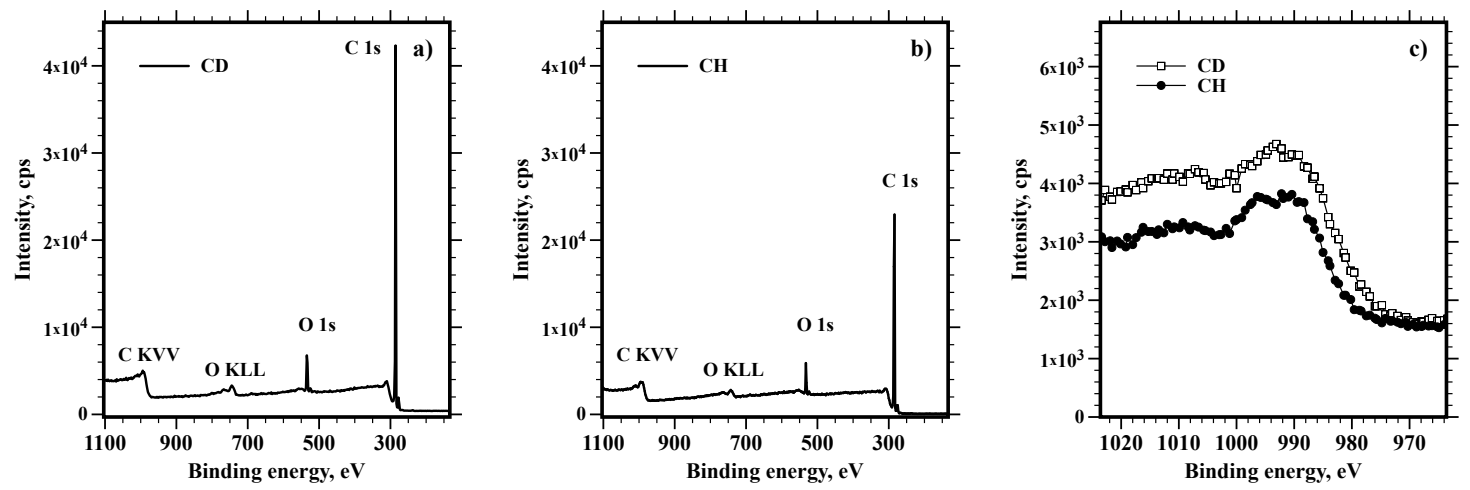

FIG. 1. XPS spectra of the hydrocarbon samples

provides information about the carbon binding structure. The shapes of the spectra for both samples are similar to graphite.

Fig. 2-a,b,c shows EPES spectra of the $\mathrm{CH}$ and $\mathrm{CD}$ samples for 3 primary electron energies: 3, 5 and $8 \mathrm{keV}$ at the scattering angle $120^{\circ}$. All spectra present a zoomed region containing the tail part of the elastic peak. The subplot in the Fig. 2-a shows the overall shape of the obtained spectra. The width of the elastic peak at half maximum is for both samples $0.8 \mathrm{eV}$ at $3 \mathrm{keV}$ and $1.2 \mathrm{eV}$ at $8 \mathrm{keV}$. The error bar depicts the relative accuracy of the instrument which is about $3 \%$. Generally, the width of the measured elastic peak is the sum of contributions from energy resolution, the energy spread of the electron gun and Doppler effect. The obtained energy resolution $(0.05 \mathrm{eV})$ is about one order of magnitude lower than the energy width of the electron beam $(0.3 \mathrm{eV}$ for $\mathrm{BaO}$ cathode), therefore it contributes only minimally to the width of the elastic peak. Instead, the Doppler broadening gives the most significant contribution for light atoms.

Elastic energy losses by electrons on carbon, protium and deuterium were calculated at the given primary energy and scattering angle from Eq. (1) and are presented as lines. It is evident that the small peaks at about $2994 \mathrm{eV}$ and $2997 \mathrm{eV}$ (Fig. 2a), $4991 \mathrm{eV}$ and $4995 \mathrm{eV}$ (Fig. 2b), $7986 \mathrm{eV}$ and $7993 \mathrm{eV}$ (Fig. 2c) correspond exactly to elastic scattering of the electrons on hydrogen isotopes. Nevertheless, Fig. 2 demonstrates that elastic energy losses can be close to the position of plasmons. It is important to point out that we have not found any significant intensity of the carbon $\pi$-plasmon despite the graphite-like shape of the Auger carbon peak. Only small intensities can be seen at Fig. 2b,c.

The energy of the $\pi$-plasmon in carbon or hydrocarbon depends on the structure and 

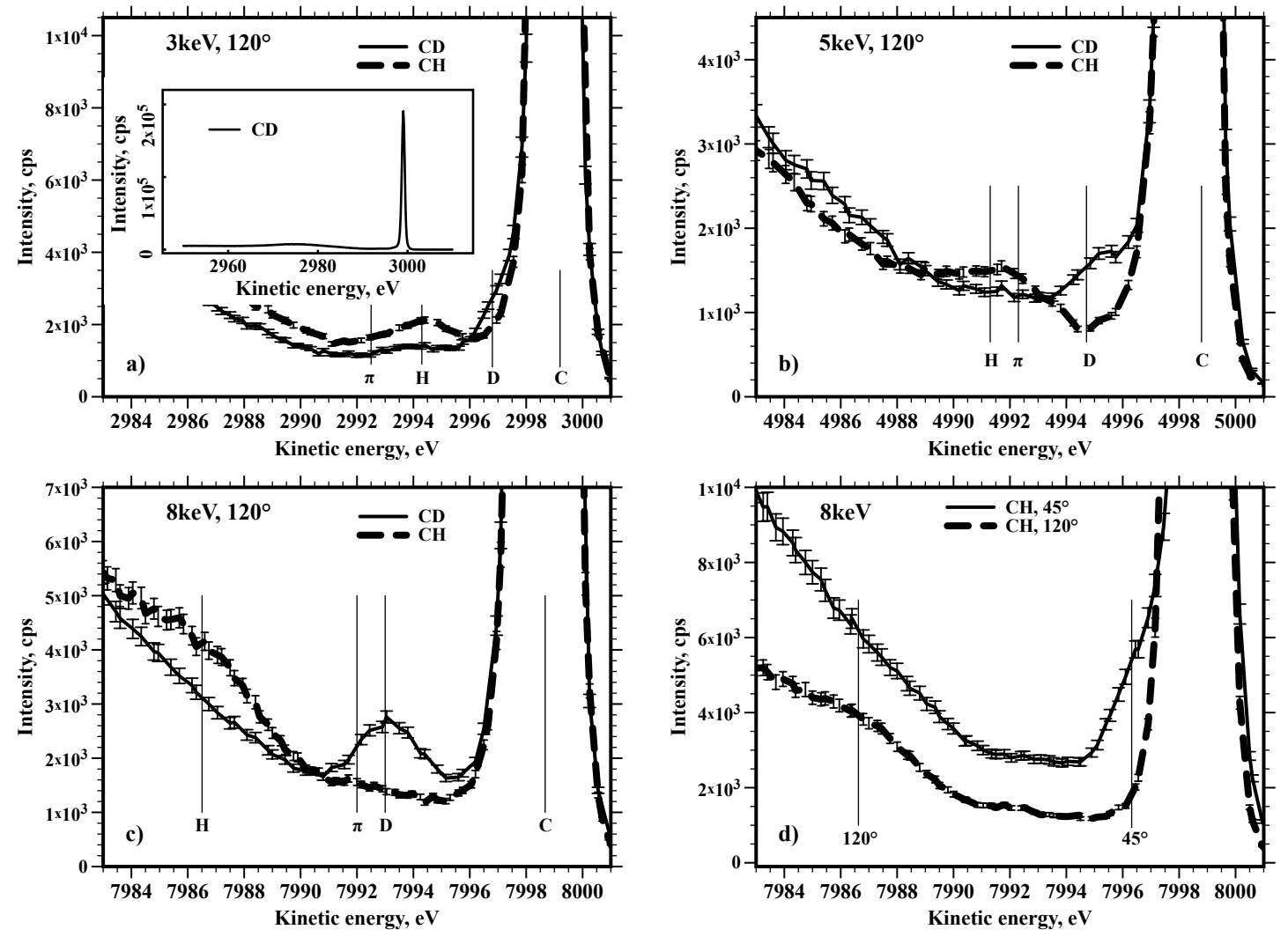

FIG. 2. EPES spectra at the different primary energies and scattering angles

chemical composition of the sample. According to the graphite-like shape of the Auger carbon peak (Fig. 1c) we took the value for the graphite $\pi$-plasmon as a reference. The energy position $(\Delta E=6.7 \mathrm{eV})$ of the graphite $\pi$ - plasmon is shown. For several energies the intensity of the $\pi$-plasmon can affect the accuracy of measuring the intensity of independent elastic peaks. Fig. 2-b,c demonstrates this situation. This problem is especially important regarding the small relative intensity of the protium elastic peak. By changing the primary energy one can clearly distinguish between two energy loss channels, because the energetic position of a plasmon is energy-independent. However, a change of the primary energy is not always possible. Occasionally, changing the scattering geometry is quite useful in this case. Fig. 2-d shows shifting of the protium elastic peak with a change of the scattering angle for the $\mathrm{CH}$ sample at a primary energy $8 \mathrm{keV}$. Additionally, changing the scattering angle can also help to verify the elemental composition. Of course, changing of the scattering geometry is limited by the geometry of the system. Nevertheless, glancing and backscattering geometries together make both quantitative and qualitative EPES analysis 
significantly clear.

Increased energy means increase of the length of the trajectory of electrons in the surface. The scanning depth can be found from Eq. 2. For carbon at $3,5,8 \mathrm{keV}$ we can estimate the scanning depth as $2.7 \mathrm{~nm}, 4.0 \mathrm{~nm}$ and $6.1 \mathrm{~nm}$ respectively. According to Kimura et al.[4] we can not consider the surface to be homogeneous at this region as it is done, for example, in NRA.

The quantitative analysis of EPES spectra is known to be relatively simple in first approximation [19]. The intensities of elastic peaks should be proportional to the differential elastic cross-section. In our case this method is not applicable for several reasons. The first reason is the unknown shape of the elastic peak and the plasmon background. The second is a significant contribution of oxygen, which is present in the XPS spectra, into the intensity of the main elastic peak. Fitting of the spectra with a simple Gaussian or Voigt line shape is possible, but is based on a large number of fitting variables, which results in several solutions for the depth profile. The shape of the elastic peak could also be more complex because of multiple elastic scattering [20]. We can conclude, that the quantitative analysis of EPES, measured with a high energy resolution, of hydrocarbon PCVD films is not trivial and is an aim for a detailed discussion. However, proper EPES interpretation can provide valuable information on the depth profile in the first $6 \mathrm{~nm}$ of the surface, which is very hard to achieve with NRA or ERD techniques.

Taken together, this work is showing for the first time the detection of deuterium and protium isotopes in the first several nanometers of PCVD films by EPES at different primary energies and scattering angles. In fusion experiments the thickness of hydrocarbon films is about $100 \mathrm{~nm}$. Thus, shown isotope sensitivity of the EPES technique could help to combine it with ion sputtering for the depth profiling. Another possibility is live analysis of the depositing films, instead of the standard Auger electron spectroscopy technique.

\section{ACKNOWLEDGEMENTS}

Sample deposition and ellipsometric measurements by K. Tichmann and T. Dürbeck are gratefully acknowledged. 
* kostanovskiyia@gmail.com

[1] J. Roth, A. Kirschner, W. Bohmeyer, S. Brezinsek, A. Cambe, E. Casarotto, R. Doerner, E. Gauthier, G. Federici, S. Higashijima, J. Hogan, A. Kallenbach, H. Kubo, J. Layet, T. Nakano, V. Philipps, A. Pospieszczyk, R. Preuss, R. Pugno, R. Ruggiéri, B. Schweer, G. Sergienko, and M. Stamp, Journal of Nuclear Materials 337-339, 970 (2005).

[2] W. Bohmeyer, A. Markin, D. Naujoks, B. Koch, G. Krenz, M. Baudach, and G. Fussmann, Journal of Nuclear Materials 363-365, 127 (2007).

[3] T. Schwarz-Selinger, A. von Keudell, and W. Jacob, Journal of Applied Physics 86, 3988 (1999).

[4] K. Kimura, K. Nakajima, S. Yamanaka, M. Hasegawa, and H. Okushi, Applied Physics Letters 78, 1679 (2001).

[5] H. Boersch, R. Wolter, and H. Schoenebeck, Zeitschrift für Physik 199, 124 (1967).

[6] M. Vos, G. P. Cornish, and E. Weigold, Review of Scientific Instruments 71, 3831 (2000).

[7] M. Vos and M. R. Went, Physical Review B 74, 205407 (2006).

[8] M. R. Went and M. Vos, Applied Physics Letters 90, 072104 (2007).

[9] B. Lesiak, A. Jablonski, Z. Prussak, and P. Mrozek, Surface science 223, 213 (1989).

[10] W. Werner, C. Tomastik, and T. Cabela, Journal of Electron Spectroscopy and Related Phenomena 113, 127 (2001).

[11] S. Tanuma, T. Shiratori, T. Kimura, K. Goto, S. Ichimura, and C. J. Powell, Surface and Interface Analysis 37, 833 (2005).

[12] S. Gurban, G. Gergely, J. Toth, D. Varga, A. Jablonski, and M. Menyhard, Surface and Interface Analysis 38, 624 (2006).

[13] W. Werner, Journal of Electron Spectroscopy and Related Phenomena 178-179, 154 (2010).

[14] F. Yubero, V. J. Rico, J. P. Espinos, J. Cotrino, and A. R. Gonzalez-Elipe, Applied Physics Letters 87, 084101 (2005).

[15] F. Yubero and K. Tkesi, Applied Physics Letters 95, 084101 (2009).

[16] G. Cooper, A. P. Hitchcock, C. A. Chatzidimitriou-Dreismann, and M. Vos, Journal of Electron Spectroscopy and Related Phenomena 155, 28 (2007). 
[17] M. Mayer, SIMNRA user's guide, Report IPP 9/113, Tech. Rep. (Max-Planck-Institut für Plasmaphysik, Garching, Germany, 1997).

[18] A. Gurbich, Nuclear Instruments and Methods in Physics Research Section B: Beam Interactions with Materials and Atoms 268, 1703 (2010).

[19] J. Zemek, J. Houdkova, B. Lesiak, A. D’Amore, D. Acierno, and L. Grassia, AIP Conference Proceedings 270, 270 (2010).

[20] V. Afanasev, M. Afanasev, A. Lubenchenko, A. Batrakov, D. Efremenko, and M. Vos, Journal of Electron Spectroscopy and Related Phenomena 177, 35 (2010). 